\title{
SARS-CoV-2 Immunoglobulin g (IgG) Kinetics in Healthcare Workers and Their Close Contacts Reduced Risk of Re-infection: South-East Asian Region
}

\author{
Mradul Kumar Daga ( $\nabla$ drmraduldaga@gmail.com ) \\ Maulana Azad Medical College https://orcid.org/0000-0001-7774-7602 \\ Govind Mawari \\ Maulana Azad Medical College \\ Vijay Kumar Karra \\ ICMR-National Institute of Malaria Research: National Institute of Malaria Research \\ Meghachandra Singh \\ Maulana Azad Medical College
}

Siddharth Chand

Maulana Azad Medical College

J Aarthi

Maulana Azad Medical College

Raghu R V

Maulana Azad Medical College

Manish Kumar Jha

Maulana Azad Medical College

Amrapali Kunwar

Maulana Azad Medical College

Sweety Mehra

Maulana Azad Medical College

\section{Research Article}

Keywords: SARS-CoV-2, immunoglobulin G (IgG), healthcare workers

Posted Date: June 2nd, 2021

DOl: https://doi.org/10.21203/rs.3.rs-540165/v1

License: (c) (1) This work is licensed under a Creative Commons Attribution 4.0 International License. Read Full License 
Page 2/17 


\section{Abstract}

\section{Background}

Severe acute respiratory syndrome coronavirus 2 (SARS-CoV-2) is responsible for global pandemic, and it has caused more than 2.3 million deaths. Persistence and stability of immunoglobulin $\mathrm{G}(\mathrm{IgG})$ response after recovery from COVID-19 infection is still uncertain.

\section{Methods}

We performed a longitudinal cohort study in healthcare workers (HCW) and their close contacts (NonHCW) with known resolved SARS-CoV-2 infection and undiagnosed infection at Maulana Azad Medical College and associated Lok Nayak hospital, New Delhi. Baseline IgG antibody titers was determined and the participants were followed over a period of six months. We also examined relationship between SARS-CoV-2 immunoglobulin G (IgG) response and new symptomatic infection in HCW and Non-HCW over time.

Results

$176(70.9 \%)$ healthcare workers and $72(29.0 \%)$ non-healthcare workers were recruited from two cohorts. 82 subjects recovered from SARS-CoV-2 infection and 166 undiagnosed for the infection having history of close contact with COVID-19 patients were followed up for a median of 227 days (interquartile range, 166 to 202) after a positive IgG antibody test. In the recovered subjects $70.7 \%$ (58) were seropositive for first anti-spike IgG assay at baseline, followed by $80.0 \%, 90.6 \%$ and $82.6 \%$ at three visits respectively. In undiagnosed subjects $37.3 \%$ (62) were seropositive at baseline, followed by $70.9 \%, 75.8 \%$ and $82.2 \%$ respectively. Also, $46.8 \%$ (29) were asymptomatic with no symptoms of COVID-19 and were seropositive at baseline. However, presence of IgG antibodies was associated with substantial reduced risk of reinfection over the follow up duration.

\section{Conclusion}

Our data showed that the antibodies levels measured increased over the first three months and decreased slightly after that and remained at a plateau and relatively stable for at least a period of six months.

\section{Introduction}

A new coronavirus disease (COVID-19), caused by SARS-CoV-2 (Severe Acute Respiratory Syndrome Coronavirus-2) has emerged as a major healthcare challenge globally (1). The clinical spectrum of the disease varies widely, ranging from asymptomatic or very mild symptoms to severe disease and death (2). Healthcare workers are at a higher risk of SARS-CoV-2 infection due to occupational exposure to infected patients and are a critical population during the current COVID-19 pandemic. 
SARS-CoV-2 attacks the respiratory system of the infected individuals, causing pneumonia and lymphopenia (3). Viral components like spike (S) and nucleocapsid $(\mathrm{N})$ proteins trigger an immune response in the host to produce immunoglobulin ( $\mathrm{IgG}$ ) to eliminate the virus (4). These viral antigens are either recognized by the B cells or are presented by major histocompatibility complex (MHC) to the T cells, resulting in antibody production, which are the main target for serological diagnosis $(4,5)$. The receptorbinding domain (RBD) in the S1 region of SARS-CoV spike (S) protein contains the critical neutralizing domain (CND), which can induce highly potent neutralizing antibody (NAbs) response and can also provide cross-protection against divergent SARS-CoV strains and is most important for vaccine development (6).

Recent evidences have shown detectable immune response towards SARS-CoV-2; however, the extent to which these neutralizing antibodies last, and the degree to which it may protect from re-infection or reduce its severity is uncertain. Despite more than 100 million people infected worldwide, re-infection of SARS-CoV-2 have been rarely reported, occurring only after mild or asymptomatic primary infection (7$11)$, suggesting that SARS-CoV-2 infection provides some immunity and protects against re-infection. In contrast, some reports suggest that humoral immune to SARS-CoV-2 infection are variable and relatively short lived (12-15). In this study, we evaluated the disease onset time, positive rate, asymptomatic infection and IgG titers against receptor-binding domain of SARS-CoV-2 in healthcare workers (HCW) who were RT-qPCR positive for SARS-CoV-2 and undiagnosed HCW and their close contacts (Non-HCW).

\section{Methods}

\section{Study Design and settings of the study}

This prospective longitudinal study was designed to: (1) check SARS-CoV-2 immunoglobulin response (IgG) after recovery from COVID-19 infection (2) explore the relationship between SARS-CoV-2 immunoglobulin response between recovered COVID-19 patients and undiagnosed individuals having close contact with COVID-19 patients over a period of six-month period. This study was carried out at the Centre for Occupational and Environmental Health, Department of Medicine, Maulana Azad Medical College, New Delhi. Healthcare workers (HCW) working within the Maulana Azad Medical College and associated Lok Nayak hospital and their close contacts (Non-HCW), active during the COVID-19 pandemic were recruited to participate in the study.

\section{Patients and plasma specimen collection}

In this study, a total of 248 subjects were recruited in two cohorts. In the first cohort, we recruited 82 patients whose clinically administered RT-qPCR test was positive and occurred before 14 days and had recovered from COVID-19 infection. The second cohort included a total of 166 undiagnosed individuals with history of contact with COVID-19 positive patients. Beginning on June 22, 2020 baseline data were collected from all the study participants. After informed consent, participants completed a baseline questionnaire with information on demographics, general health, recent symptoms, occupation, and potential COVID-19 exposure followed by plasma specimen collection in ethylene diamine tetraacetic acid 
anticoagulant tube. All study participants were longitudinally followed for up to six months and plasma samples were collected after every two months, every participant has four visits to the clinic.

\section{Enzyme-linked immunosorbent assay (ELISA) for SARS-CoV-2 IgG antibody}

The presences of antibodies against SARS-CoV-2 were examined by enzyme linked immunosorbent assay (ELISA) ErbaLisa ${ }^{\circledR}$ COVID-19 lgG semi quantitative kit (Erba Mannheim and Calbiotech, U.S.A) according to manufacturer's instructions. This kit was developed utilizing recombinant proteins of SARSCoV-2 i.e., Spike-2 and nucleoprotein for detection of anti-COVID-19 antibodies. ELISAs cut-off values as per the protocol were as follows: the optical density (OD) values of the negative controls (between 0.19 and 0.22 ) were averaged and adjusted by addition of a constant (0.18) and the resultant reference value was multiplied by a correction factor of 1.1 which was the cut-off value; any value above this was considered positive and below was negative.

\section{Statistical analysis}

The categorical variables were calculated as frequencies and percentages, and continuous variables were described using the median with interquartile range (IQR). Statistical relationship between the groups was calculated using Chi-square test and Fisher's exact test for categorical variables, Kruskal Wallis $\mathrm{H}$ test for comparison of IgG titers. $P<0.05$ was considered statistically significant. All Statistical analysis were conducted using SPSS 25.

\section{Results}

\section{The demographic and clinical information}

A total of 248 subjects were recruited from two cohorts, in which 174 (70.2\%) were males. The subjects were aged from 5 to 80 years (mean 40.3). Based on their working environment, they were classified into HCW $(176,70.9 \%)$ and Non-HCW $(72,29.0 \%)$. HCW were enrolled from COVID-19 medicine units, COVID19 ICUs, specialty consultants, and pharmacies. These HCW had different degree of workplace exposures, performing invasive bedside procedures, providing intraoperative and routine care to COVID-19 positive patients and working in pharmacies. The Non-HCW enrolled were the close contacts of HCW. The first cohort consisted of HCW $(68.3 \%, 56 / 82)$ and Non-HCW $(31.7 \%, 26 / 82)$ recovered from COVID-19 infection, and the second cohort consisted of HCW $(72.3 \%, 120 / 166)$ and Non-HCW $(27.7 \%, 46 / 166)$ undiagnosed for COVID-19 infection. The majority of the patients recalled having clinical symptoms consistent with COVID-19, including fever $(122,49.2 \%)$, fatigue $(93,37.5 \%)$, muscle pain $(78,31.5 \%)$, cough $(58,23.4 \%)$ and throat pain $(58,23.4 \%)$ (Table 1, and Figure 2). Comorbidities were reported in 106 $(42.7 \%)$ patients, in the form of hypertension, diabetes, thyroid, asthma, cardiovascular disease, COPD etc (Table 1). Compared to undiagnosed for COVID-19 cases, the disease symptoms including fever $(75.6 \%$ vs $36.1 \%)(P=<0.001)$, cough (40.2\% vs $15.1 \%)(P=<0.001)$, fatigue (61.0\% vs $25.9 \%)(P=<0.001)$, breathlessness ( $17.1 \%$ vs $5.4 \%)(P=0.002)$, muscle pain ( $42.7 \%$ vs $25.9 \%)(P=0.007)$, loss of appetite (26.8\% vs $10.2 \%)(P=<0.001)$, altered smell $(37.8 \%$ vs $4.8 \%)(P=<0.001)$, and loss of taste $(41.5 \%$ vs 
10.2\%) $(P=<0.001)$ were reported in higher frequency in COVID-19 positive cases (chi-square test) (Table 1 , and Figure 2).

\section{Characteristics of IgG antibodies in the study population}

A total of 659 plasma samples were collected from the 248 study participants, In the first cohort, 82 patients recovered from COVID-19 infection provided 82 plasma samples at baseline visit and out of them 75 patients were followed up longitudinally for six months (three visits) and provided 307 plasma samples (the time interval between each visit is 45 days). In the second cohort, 166 undiagnosed subjects for COVID-19, with history of close contact with COVID-19 positive patients provided 166 plasma samples at baseline visit and out of them 62 patients with seropositive for anti-spike IgG assay were followed up longitudinally for six months (three visits) and provided 352 plasma samples, respectively. HCW and NonHCW were followed for a median of 227 days (interquartile range, 166 to 202) after a positive antibody test.

The IgG antibodies against spike (S) protein were tested in 248 subjects at baseline visit, 137 samples at $1^{\text {st }}, 2^{\text {nd }}$ and $3^{\text {rd }}$ visits, respectively. All the serum samples were collected between 1 and 238 days after onset of symptom (The number of days between visits was calculated from the date of symptom onset in the symptomatic COVID-19 positive individuals and the date of the first clinic attendance in the asymptomatic undiagnosed individuals with no PCR performed). IgG antibodies appeared from day 16 and remained stable during the course. In the first cohort, 70.7\% (58/82) subjects were seropositive at their first anti-spike IgG assay at baseline visit, seroconversion occurred during the study period and $80.0 \%(60 / 75)$ by $1^{\text {st }}$ visit, $90.6 \%(68 / 75)$ by $2^{\text {nd }}$ visit and finally $82.6 \%(62 / 75)$ seropositive at $3^{\text {rd }}$ visit have seroconverted. Similarly, in the second cohort, 37.3\% (62/166) subjects were seropositive at their first anti-spike IgG assay at baseline visit. These 62 seropositive subjects were followed up and were looked for immune response; by the $1^{\text {st }}$ visit $70.9 \%(44 / 62), 75.8 \%(47 / 62)$ by $2^{\text {nd }}$ visit and finally $82.2 \%$ $(51 / 62)$ were found to be seropositive. In the second cohort, $46.8 \%(29 / 62)$ subjects were asymptomatic, having no COVID-19 symptoms and were seropositive for anti-spike IgG at baseline visit. The positivity rates of IgG antibodies increased over time and remained stable across the study duration (Figure 1).

\section{SARS-CoV-2 antibody titers in COVID-19 patients}

The antibodies levels measured increased over the first three months and decreased slightly after that and remained at a plateau during the entire study duration. In COVID-19 positive cohort, we found a significant difference between IgG antibody levels at weeks 1-3 (2.14 \pm 1.98$)$, weeks 3-7 (2.55 \pm 1.55$)$, weeks

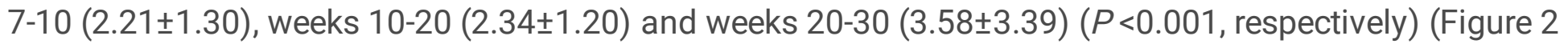
B). In the undiagnosed cohort, 76.3\% (29/38) patients were asymptomatic at baseline visit (weeks 1-3) with IgG antibody levels (2.26 \pm 1.32$)$. IgG antibody levels were also found to be significantly different in

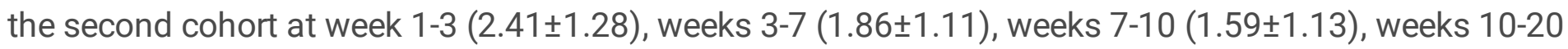
$(2.10 \pm 1.16)$ and weeks $20-30(3.27 \pm 2.59)(P<0.001$, respectively). The tabulated data of IgG antibody titers by their visits were shown in the Table 2 and Figure 1. 
We then sought to evaluate the duration of appearance of IgG antibodies and their levels during the course of the disease. We observed the kinetics of the antibody levels remained mostly similar at all the four visits i.e., up to six months. The IgG antibodies started to appeared between weeks 1-3 and increased gradually and remained stable during the disease course. In the COVID-19 positive cohort, we found $70.7 \%$ (58/82) PCR positive and IgG antibody positive cases with significant difference in antibody titers $3.21 \pm 1.28$ at baseline visit, $2.53 \pm 0.73$ at visit $1,2.82 \pm 1.41$ at visit 2 , and $4.32 \pm 3.75$ at visit $3(P<0.001$, respectively) (Table 2). In the same cohort, $29.2 \%$ (24/82) cases were PCR positive and IgG antibody negative at the baseline, the $\lg G$ antibody levels were very low throughout the course of study with antibody titers $0.66 \pm 0.23$ at baseline visit, $0.68 \pm 0.25$ at visit $1,0.53 \pm 0.33$ at visit 2 , and $0.84 \pm 0.25$ at visit 3 , $(P=0.054$, respectively) (Table 2$)$. In the undiagnosed cohort, $37.3 \%(62 / 166)$ cases were found to be IgG positive at the baseline, and IgG antibody titers remained to be mostly similar in these cases throughout the course of study with antibody titers $2.24 \pm 1.28$ at baseline visit, $2.10 \pm 0.77$ at visit 1 , $2.54 \pm 1.02$ at visit 2 , and $3.64 \pm 2.56$ at visit $3,(P<0.001$, respectively) (Table 2$)$. In the same cohort, $46.7 \%$ $(29 / 62)$ cases had developed IgG antibodies at the baseline, these cases were asymptomatic IgG antibody titers were initially low in these cases but increase gradually during the course of study with antibody titers $1.91 \pm 1.46$ at baseline visit, $1.88 \pm 0.93$ at visit $1,2.38 \pm 1.35$ at visit 2 , and $3.24 \pm 2.68$ at visit 3 , ( $P=0.021$, respectively) (Table 2$)$. Further, $53.2 \%$ (33/62) cases had history of COVID-19 symptoms, similarly IgG antibody titers were initially low but increase gradually during the course of study with antibody titers $1.98 \pm 1.37$ at baseline visit, $1.79 \pm 0.94$ at visit $1,2.09 \pm 1.08$ at visit 2 , and $3.48 \pm 0.46$ at visit 3 , $(P<0.001$, respectively) (Table 2$)$.

\section{Discussion}

In our longitudinal cohort study, among 248 study participants $68.3 \%$ of healthcare workers with previous diagnosis of SARS-CoV-2-positive and $72.3 \%$ of healthcare workers in participants without previous infection shows that the healthcare workers are at the highest risk of the SARS-CoV-2 infection. In addition, presence of $46.8 \%$ asymptomatic infection in the undiagnosed patients strengthen the speculation among the healthcare providers, researchers and policy makers that asymptomatic infections plays a major role in the spread of SARS-CoV-2 infection (16). In a recent study on presymptomatic SARSCoV-2 Infections and transmission in a skilled nursing facility showed $56 \%$ asymptomatic patients at the time of testing and also highlighted that asymptomatic and/or presymptomatic infections certainly played a role in disease dissemination (16), these findings are consistent with our results in which $86.2 \%$ asymptomatic healthcare workers developed IgG antibodies at baseline visit showing that healthcare workers are previously infected with SARS-CoV-2. Therefore, a better understanding of SARS-CoV-2 dynamics in healthcare workers is imperative in protecting this key workforce and also reducing their role in nosocomial infection and community spread.

Importantly, the presence of anti-spike IgG antibodies shows the previous infection and is associated with protection from re-infection. In our study, in patients recovered from COVID-19 infection anti-spike IgG antibodies developed in $70.7 \%$ at baseline visit and $82.6 \%$ at $3^{\text {rd }}$ visit, over the 32 -week follow-up. We also 
observed stable antibody titers over a period of at least three months and only modest declines at the sixmonth time point, which is consistent with study carried out by Ania et al., at Mount Sinai Health System in New York City and other studies (17-23). Furthermore, the presence of these antibodies was associated with substantial reduced risk of re-infection over the follow up duration. Only three PCRpositive results were reported in the asymptomatic healthcare workers with IgG antibodies, suggesting that IgG antibodies developed against previous infection is associated with protection towards reinfection in most of the subjects for at least six months. Also, seroreversion was observed only in small percentage of patients. However, it is noted that non hospitalized patients with mild symptoms have lower levels of antibody titers compared to hospitalized patients with severe illness (24-26). The differences in IgG antibody titers between participants may be associated with difference in antigen exposure. However, looking at the lower number of re-infection rate in seropositive individuals, we can assume these antibody titers regulate protection from infection. Though, it is still not known that how long these antibodies will provide protection towards SARS-CoV-2 infection, but we know from the previous serological studies on Middle East Respiratory Syndrome Coronavirus (MERS) and SARS-CoV infection (responsible for 2003 epidemic) that these neutralizing antibodies are relatively short lived and can last for three years (27-30). Additionally, recent evidences from the nonhuman primate models shows that SARS- CoV-2 infection does provide protection from re-infection for at least some time $(31,32)$. Studies also proved that transferring monoclonal antibodies or serum from convalescent animals to naïve animals produce protective antibodies and reduces virus replication significantly $(33,34)$.

There are some limitations in the study. The samples size was small and study duration was relatively short up to 32 weeks of follow up. Healthcare workers and Non-healthcare workers were recruited in a voluntary testing basis with a flexible follow-up schedule, which resulted in different attendance frequencies. In addition, as the healthcare workers were told their baseline antibody results, some seropositive staff were reluctant to come for follow-up. The longitudinal follow-up of the same individual will generate accurate data and more robust kinetics of antibody responses. We are planning to follow these cohorts over longer intervals of time. We believe that ongoing follow-up and further investigations, using humoral and cellular immunity markers involving large scale of clinical samples will provide better understanding of the antibody kinetics and information on significance, magnitude and duration of protection from re-infection, causing symptomatic disease, hospitalization or death with SARS-CoV-2 infection.

\section{Declarations}

- Ethics approval: This study was conducted only after approval from the institutional ethical committee Maulana Azad Medical College \& with the aid of informed consent from all the patient participants.

- Funding: No organised funding source was used in study conduction.

- Acknowledgements: None 
- Conflict of interests: The authors declare that they have no conflict of interest.

\section{References}

1. LI R, Ym W, Zq W, Zc X, L G, T X, et al. Identification of a novel coronavirus causing severe pneumonia in human: a descriptive study [Internet]. Chinese medical journal. 2020 [cited 2021 Jan 27]. Available from: https://pubmed.ncbi.nlm.nih.gov/32004165/

2. L R, L Z, D C, J W, Y H, H C, et al. The kinetics of humoral response and its relationship with the disease severity in COVID-19 [Internet]. Communications biology. 2020 [cited 2021 Jan 27]. Available from: https://pubmed.ncbi.nlm.nih.gov/33311543/

3. Zhang Y, Geng X, Tan Y, Li Q, Xu C, Xu J, et al. New understanding of the damage of SARS-CoV-2 infection outside the respiratory system. Biomed Pharmacother. 2020 Jul;127:110195.

4. Dhama K, Sharun K, Tiwari R, Dadar M, Malik YS, Singh KP, et al. COVID-19, an emerging coronavirus infection: advances and prospects in designing and developing vaccines, immunotherapeutics, and therapeutics. Hum Vaccines Immunother. 2020 Mar 18;1-7.

5. L G, L R, S Y, M X, D C, F Y, et al. Profiling Early Humoral Response to Diagnose Novel Coronavirus Disease (COVID-19) [Internet]. Clinical infectious diseases: an official publication of the Infectious Diseases Society of America. 2020 [cited 2021 Jan 27]. Available from: https://pubmed.ncbi.nlm.nih.gov/32198501/

6. Zhu X, Liu Q, Du L, Lu L, Jiang S. Receptor-binding domain as a target for developing SARS vaccines. J Thorac Dis. 2013 Aug;5(Suppl 2):S142-S148.

7. Sf L, D O, Ne S, Pc M, A H, Sb H, et al. Antibody Status and Incidence of SARS-CoV-2 Infection in Health Care Workers [Internet]. The New England journal of medicine. 2020 [cited 2021 Jan 27]. Available from: https://pubmed.ncbi.nlm.nih.gov/33369366/

8. Tillett RL, Sevinsky JR, Hartley PD, Kerwin H, Crawford N, Gorzalski A, et al. Genomic evidence for reinfection with SARS-CoV-2: a case study. Lancet Infect Dis. 2021 Jan;21(1):52-8.

9. Van Elslande J, Vermeersch P, Vandervoort K, Wawina-Bokalanga T, Vanmechelen B, Wollants E, et al. Symptomatic SARS-CoV-2 reinfection by a phylogenetically distinct strain. Clin Infect Dis [Internet]. [cited 2021 Jan 27]; Available from: https://academic.oup.com/cid/advancearticle/doi/10.1093/cid/ciaa1330/5901661

10. Kk T, If H, Jd I, Aw C, Wm C, Ar T, et al. COVID-19 re-infection by a phylogenetically distinct SARScoronavirus-2 strain confirmed by whole genome sequencing [Internet]. Clinical infectious diseases: an official publication of the Infectious Diseases Society of America. 2020 [cited 2021 Jan 27]. Available from: https://pubmed.ncbi.nlm.nih.gov/32840608/

11. V G, Rc B, A J, S S, R U, M I, et al. Asymptomatic reinfection in two healthcare workers from India with genetically distinct SARS-CoV-2 [Internet]. Clinical infectious diseases: an official publication of the Infectious Diseases Society of America. 2020 [cited 2021 Jan 27]. Available from: https://pubmed.ncbi.nlm.nih.gov/32964927/ 
12. N K, S B, T S. Duration of anti-SARS-CoV-2 antibodies much shorter in India [Internet]. Vaccine. 2021 [cited 2021 Jan 27]. Available from: https://pubmed.ncbi.nlm.nih.gov/33189428/

13. $X W, X G, Q X, Y P, Y H, J$ L, et al. Neutralizing Antibodies Responses to SARS-CoV-2 in COVID-19 Inpatients and Convalescent Patients [Internet]. Clinical infectious diseases: an official publication of the Infectious Diseases Society of America. 2020 [cited 2021 Jan 27]. Available from: https://pubmed.ncbi.nlm.nih.gov/32497196/

14. Qx L, Xj T, QI S, Q L, Hj D, J Y, et al. Clinical and immunological assessment of asymptomatic SARSCoV-2 infections [Internet]. Nature medicine. 2020 [cited 2021 Jan 27]. Available from: https://pubmed.ncbi.nlm.nih.gov/32555424/

15. Ms S, Mg Z, Rc K, G M, SI L, Wh H, et al. Rapid Generation of Neutralizing Antibody Responses in COVID-19 Patients [Internet]. Cell reports. Medicine. 2020 [cited 2021 Jan 27]. Available from: https://pubmed.ncbi.nlm.nih.gov/32835303/

16. Mm A, Km H, Sc R, A K, A J, Jr J, et al. Presymptomatic SARS-CoV-2 Infections and Transmission in a Skilled Nursing Facility [Internet]. The New England journal of medicine. 2020 [cited 2021 Feb 1]. Available from: https://pubmed.ncbi.nlm.nih.gov/32329971/

17. A W, F A, A F, Dr A, Mj B, M M, et al. Robust neutralizing antibodies to SARS-CoV-2 infection persist for months [Internet]. Science (New York, N.Y.). 2020 [cited 2021 Feb 2]. Available from: https://pubmed.ncbi.nlm.nih.gov/33115920/

18. F M, H W, B B, M S, E S, C R, et al. Longitudinal analysis of serology and neutralizing antibody levels in COVID19 convalescents [Internet]. The Journal of infectious diseases. 2020 [cited 2021 Feb 2]. Available from: https://pubmed.ncbi.nlm.nih.gov/33140086/

19. J S, C G, B M, S A, S P, Kja S, et al. Longitudinal observation and decline of neutralizing antibody responses in the three months following SARS-CoV-2 infection in humans [Internet]. Nature microbiology. 2020 [cited 2021 Feb 2]. Available from: https://pubmed.ncbi.nlm.nih.gov/33106674/

20. G B-B, A L, Sp A, J P, R G, G G, et al. Decline of Humoral Responses against SARS-CoV-2 Spike in Convalescent Individuals [Internet]. mBio. 2020 [cited 2021 Feb 2]. Available from: https://pubmed.ncbi.nlm.nih.gov/33067385/

21. Khd $C, A s D, R E, C r W, N W$, Jk L, et al. Dynamics of neutralizing antibody titers in the months after SARS-CoV-2 infection [Internet]. The Journal of infectious diseases. 2020 [cited 2021 Feb 2]. Available from: https://pubmed.ncbi.nlm.nih.gov/33000143/

22. As I, Fk J, A N, M K, M B, D S, et al. Persistence and decay of human antibody responses to the receptor binding domain of SARS-CoV-2 spike protein in COVID-19 patients [Internet]. Science immunology. 2020 [cited 2021 Feb 2]. Available from: https://pubmed.ncbi.nlm.nih.gov/33033172/

23. $K$ W, Qx L, Hj D, J H, Qz G, Gj Z, et al. Longitudinal dynamics of the neutralizing antibody response to SARS-CoV-2 infection [Internet]. Clinical infectious diseases: an official publication of the Infectious Diseases Society of America. 2020 [cited 2021 Feb 2]. Available from: https://pubmed.ncbi.nlm.nih.gov/32745196/ 
24. C C, J N, Y Z, A V, J S, A W, et al. Systemic and mucosal antibody responses specific to SARS-CoV-2 during mild versus severe COVID-19 [Internet]. The Journal of allergy and clinical immunology. 2020 [cited 2021 Feb 2]. Available from: https://pubmed.ncbi.nlm.nih.gov/33221383/

25. M D, L K, L P, C G, M Y, R H, et al. Novel SARS-CoV-2 specific antibody and neutralization assays reveal wide range of humoral immune response during COVID-19 [Internet]. medRxiv : the preprint server for health sciences. 2020 [cited 2021 Feb 2]. Available from: https://pubmed.ncbi.nlm.nih.gov/32676617/

26. SI K, A P, Hs P, RI U, Jr S, Se B, et al. Sex, age, and hospitalization drive antibody responses in a COVID-19 convalescent plasma donor population [Internet]. The Journal of clinical investigation. 2020 [cited 2021 Feb 2]. Available from: https://pubmed.ncbi.nlm.nih.gov/32764200/

27. Dc P, I I, B R, S A, A H, M AN, et al. Persistence of Antibodies against Middle East Respiratory Syndrome Coronavirus [Internet]. Emerging infectious diseases. 2016 [cited 2021 Feb 2]. Available from: https://pubmed.ncbi.nlm.nih.gov/27332149/

28. W L, A F, Ph Z, L Z, Zt X, L B, et al. Two-year prospective study of the humoral immune response of patients with severe acute respiratory syndrome [Internet]. The Journal of infectious diseases. 2006 [cited 2021 Feb 2]. Available from: https://pubmed.ncbi.nlm.nih.gov/16479513/

29. Lp W, Nc W, Yh C, Xy T, Dy N, Ly Z, et al. Duration of antibody responses after severe acute respiratory syndrome [Internet]. Emerging infectious diseases. 2007 [cited 2021 Feb 2]. Available from: https://pubmed.ncbi.nlm.nih.gov/18258008/

30. F T, Y Q, Zt X, J W, Mj M, H L, et al. Lack of peripheral memory B cell responses in recovered patients with severe acute respiratory syndrome: a six-year follow-up study [Internet]. Journal of immunology (Baltimore, Md.: 1950). 2011 [cited 2021 Feb 2]. Available from: https://pubmed.ncbi.nlm.nih.gov/21576510/

31. A C, J L, Aj M, K M, Nb M, L P, et al. SARS-CoV-2 infection protects against rechallenge in rhesus macaques [Internet]. Science (New York, N.Y.). 2020 [cited 2021 Feb 2]. Available from: https://pubmed.ncbi.nlm.nih.gov/32434946/

32. W D, L B, J L, C X, J L, J X, et al. Primary exposure to SARS-CoV-2 protects against reinfection in rhesus macaques [Internet]. Science (New York, N.Y.). 2020 [cited 2021 Feb 2]. Available from: https://pubmed.ncbi.nlm.nih.gov/32616673/

33. M I, K I-H, M H, S L, Pj H, N N, et al. Syrian hamsters as a small animal model for SARS-CoV-2 infection and countermeasure development [Internet]. Proceedings of the National Academy of Sciences of the United States of America. 2020 [cited 2021 Feb 2]. Available from: https://pubmed.ncbi.nlm.nih.gov/32571934/

34. R S, C S, X D, Z C, P L, J S, et al. A human neutralizing antibody targets the receptor-binding site of SARS-CoV-2 [Internet]. Nature. 2020 [cited 2021 Feb 2]. Available from: https://pubmed.ncbi.nlm.nih.gov/32454512/

\section{Tables}


Table 1: Demographic and clinical characteristics of study participants. 


\begin{tabular}{|c|c|c|c|c|}
\hline $\begin{array}{l}\text { Demographic and clinical } \\
\text { variables }\end{array}$ & $\begin{array}{l}\text { Total } \\
(n=248)\end{array}$ & $\begin{array}{l}\text { SARS-CoV-2 RT-qPCR } \\
\text { positive }(n=82)\end{array}$ & $\begin{array}{l}\text { Not tested for } \\
\text { SARS-CoV-2 } \\
(n=166)\end{array}$ & $\begin{array}{l}P \\
\text { value }\end{array}$ \\
\hline \multicolumn{5}{|l|}{ Sex - no (\%) } \\
\hline Male & $\begin{array}{l}174 \\
(70.2)\end{array}$ & $58(70.7)$ & $116(69.9)$ & 0.890 \\
\hline Female & $\begin{array}{l}74 \\
(29.8)\end{array}$ & $24(29.3)$ & $50(30.1)$ & \\
\hline Mean age (+/-SD)-yr & $\begin{array}{l}40.3 \\
(13.0)\end{array}$ & $38.9(12.3)$ & $40.8(13.3)$ & 0.279 \\
\hline Healthcare workers & $\begin{array}{l}176 \\
(70.9)\end{array}$ & $56(68.3)$ & $120(72.3)$ & \\
\hline Physician & $\begin{array}{l}49 \\
(19.8)\end{array}$ & $14(17.0)$ & $35(21.1)$ & 0.028 \\
\hline Nurse & $21(8.5)$ & $8(9.8)$ & $13(7.8)$ & \\
\hline Nursing orderly & $\begin{array}{l}63 \\
(25.4)\end{array}$ & $14(17.0)$ & $49(29.5)$ & \\
\hline Lab Technician & $21(8.5)$ & $7(8.5)$ & $14(8.4)$ & \\
\hline Pharmacist & $22(8.9)$ & $13(15.9)$ & $9(5.4)$ & \\
\hline Non healthcare worker & $\begin{array}{l}72 \\
(29.0)\end{array}$ & $26(31.7)$ & $46(27.7)$ & \\
\hline Businessman & $23(9.3)$ & $11(13.4)$ & $12(7.2)$ & 0.259 \\
\hline Housewife & $\begin{array}{l}25 \\
(10.1)\end{array}$ & $10(12.2)$ & $15(9.0)$ & \\
\hline Police & $13(5.2)$ & $3(3.7)$ & $10(6.0)$ & \\
\hline Student & $11(4.4)$ & $2(2.4)$ & $9(5.4)$ & \\
\hline \multicolumn{5}{|l|}{$\begin{array}{l}\text { Typical COVID-19 symptoms } \\
\text {-no (\%) }\end{array}$} \\
\hline \multicolumn{5}{|l|}{ Respiratory symptoms } \\
\hline Cough & $\begin{array}{l}58 \\
(23.4)\end{array}$ & $33(40.2)$ & $25(15.1)$ & $<.001$ \\
\hline Breathlessness & $23(9.3)$ & $14(17.1)$ & $9(5.4)$ & $<.001$ \\
\hline Fatigue & $\begin{array}{l}93 \\
(37.5)\end{array}$ & $50(61.0)$ & $43(25.9)$ & $<.001$ \\
\hline Throat pain & $\begin{array}{l}58 \\
(23.4)\end{array}$ & $23(28.0)$ & $35(21.1)$ & 0.222 \\
\hline
\end{tabular}


Systemic symptoms

\begin{tabular}{|c|c|c|c|c|}
\hline Fever & $\begin{array}{l}122 \\
(49.2)\end{array}$ & $62(75.6)$ & $60(36.1)$ & $<.001$ \\
\hline Cold & $\begin{array}{l}44 \\
(17.7)\end{array}$ & $15(18.3)$ & $29(17.1)$ & 0.873 \\
\hline Headache & $\begin{array}{l}50 \\
(20.2)\end{array}$ & $21(25.6)$ & $29(17.1)$ & 0.132 \\
\hline Muscle pain & $\begin{array}{l}78 \\
(31.5)\end{array}$ & $35(42.7)$ & $43(25.9)$ & $<0.001$ \\
\hline Loss of appetite & $\begin{array}{l}39 \\
(15.7)\end{array}$ & $22(26.8)$ & $17(10.2)$ & $<0.001$ \\
\hline Diarrhoea & $\begin{array}{l}27 \\
(10.9)\end{array}$ & $12(14.6)$ & $15(9.0)$ & 0.183 \\
\hline Vomiting & $10(4.0)$ & $5(6.1)$ & $5(3.0)$ & 0.245 \\
\hline Altered smell & $\begin{array}{l}39 \\
(15.7)\end{array}$ & $31(37.8)$ & $8(4.8)$ & $<0.001$ \\
\hline Loss of taste & $\begin{array}{l}51 \\
(20.6)\end{array}$ & $34(41.5)$ & $17(10.2)$ & $<0.001$ \\
\hline No symptoms & $\begin{array}{l}29 \\
(11.6)\end{array}$ & - & $29(17.4)$ & \\
\hline \multicolumn{5}{|l|}{ Co morbidities } \\
\hline Hypertension & $\begin{array}{l}36 \\
(14.5)\end{array}$ & $10(12.1)$ & $26(15.6)$ & 0.465 \\
\hline Asthma & $8(3.2)$ & $1(1.2)$ & $7(4.2)$ & 0.208 \\
\hline COPD & $2(0.8)$ & $1(1.2)$ & $1(0.6)$ & 0.609 \\
\hline Cardiovascular disease & $3(1.2)$ & $2(2.4)$ & $1(0.6)$ & 0.213 \\
\hline Rheumatoid arthritis & $3(1.2)$ & $1(1.2)$ & $2(1.2)$ & 0.992 \\
\hline Cancer & $2(0.8)$ & $1(1.2)$ & $1(0.6)$ & 0.609 \\
\hline Thyroid & $16(6.5)$ & $5(6.1)$ & $11(6.6)$ & 0.873 \\
\hline Diabetes & $22(8.9)$ & $9(11.0)$ & $13(7.8)$ & 0.412 \\
\hline Liver disease & $4(1.6)$ & $3(3.7)$ & $1(0.6)$ & 0.072 \\
\hline Kidney disease & - & - & - & - \\
\hline TB & $4(1.6)$ & $2(2.4)$ & $2(1.2)$ & 0.467 \\
\hline HIV & - & - & - & \\
\hline
\end{tabular}




\begin{tabular}{|lllll|} 
Neurological disorder & $3(1.2)$ & - & $3(1.8)$ & - \\
Osteoarthritis & $2(0.8$ & - & $2(1.2)$ & - \\
\hline Anemia & $1(0.4)$ & $1(1.2)$ & - & - \\
\hline
\end{tabular}

Table 2: IgG ELISA optical densities of the study participants for SARS-CoV-2

\begin{tabular}{|c|c|c|c|c|c|c|}
\hline \multirow[t]{3}{*}{ SARS-CoV-2 test results } & \multirow[t]{3}{*}{$\begin{array}{l}\text { Total } \\
(\mathrm{N}=248)\end{array}$} & $\begin{array}{l}\text { Mean } \\
\text { (SD) }\end{array}$ & $\begin{array}{l}\text { Mean } \\
\text { (SD) }\end{array}$ & $\begin{array}{l}\text { Mean } \\
\text { (SD) }\end{array}$ & $\begin{array}{l}\text { Mean } \\
\text { (SD) }\end{array}$ & \multirow[t]{3}{*}{$\begin{array}{l}\text { P- } \\
\text { value* }\end{array}$} \\
\hline & & IgG Titer & IgG Titer & IgG Titer & IgG Titer & \\
\hline & & Baseline & Visit \#1 & Visit \#2 & Visit \#3 & \\
\hline \multicolumn{7}{|l|}{$\begin{array}{l}\text { SARS-CoV-2 RT qPCR } \\
\text { positive }\end{array}$} \\
\hline PCR Positive-no. (\%) & 82 & $2.46 \pm 1.59$ & $2.16 \pm 0.99$ & $2.61 \pm 1.50$ & $3.71 \pm 3.66$ & $<.0001$ \\
\hline $\begin{array}{l}\text { PCR Positive / IgG } \\
\text { Positive-no. (\%) }\end{array}$ & 58 & $3.21 \pm 1.28$ & $2.53 \pm 0.73$ & $2.82 \pm 1.41$ & $4.32 \pm 3.75$ & $<.0001$ \\
\hline $\begin{array}{l}\text { PCR positive / IgG } \\
\text { Negative-no. (\%) }\end{array}$ & 24 & $0.66 \pm 0.23$ & $0.68 \pm 0.25$ & $0.53 \pm 0.33$ & $0.84 \pm 0.25$ & 0.054 \\
\hline \multicolumn{7}{|l|}{$\begin{array}{l}\text { SARS-CoV-2 PCR test } \\
\text { not done }\end{array}$} \\
\hline Total tested & 166 & $1.10 \pm 1.15$ & $1.69 \pm 0.97$ & $2.10 \pm 1.22$ & $3.34 \pm 2.60$ & $<.0001$ \\
\hline IgG Positive-no. (\%) & 62 & $2.24 \pm 1.28$ & $2.10 \pm 0.77$ & $2.54 \pm 1.02$ & $3.64 \pm 2.56$ & $<.0001$ \\
\hline $\begin{array}{l}\text { Asymptomatic patients- } \\
\text { no. (\%) }\end{array}$ & 29 & $1.91 \pm 1.46$ & $1.88 \pm 0.93$ & $2.38 \pm 1.35$ & $3.24 \pm 2.68$ & 0.021 \\
\hline $\begin{array}{l}\text { Previous history of } \\
\text { COVID-19 symptoms-no. } \\
\text { (\%) }\end{array}$ & 33 & $1.98 \pm 1.37$ & $1.79 \pm 0.94$ & $2.09 \pm 1.08$ & $3.48 \pm 0.46$ & $<.0001$ \\
\hline
\end{tabular}

* $P$-values calculated using Kruskal Wallis $\mathrm{H}$ test for comparing multiple groups.

\section{Figures}




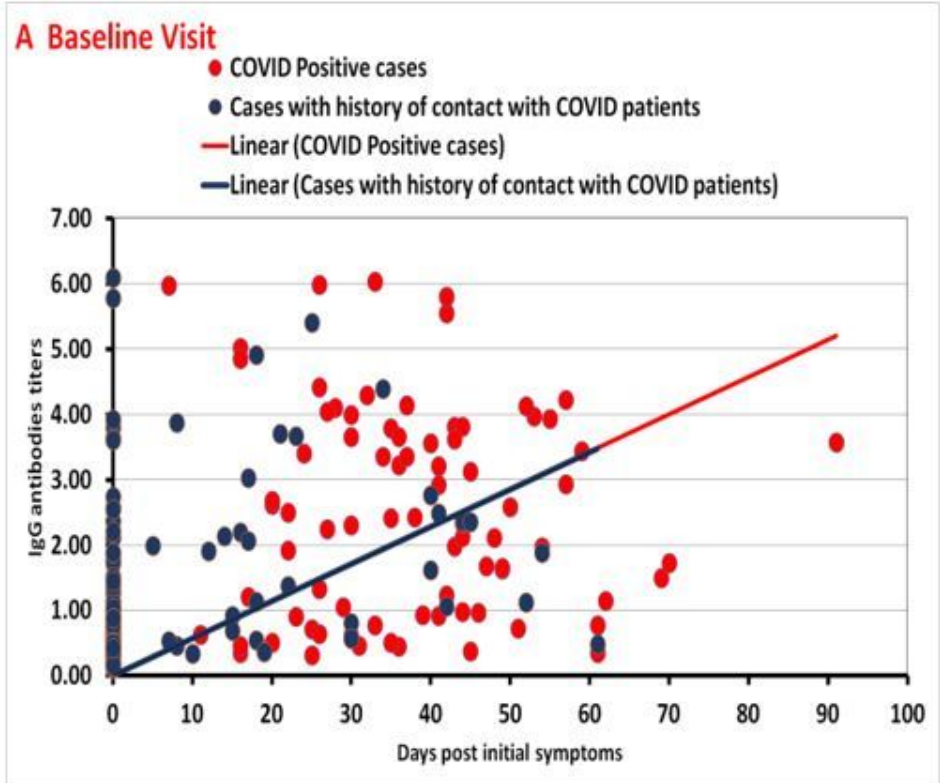

B Visit\#1 - COVID Positive cases

- Cases with history of contact with COVID patients

- Linear (COVID Positive cases)

- Linear (Cases with history of contact with COVID patients)
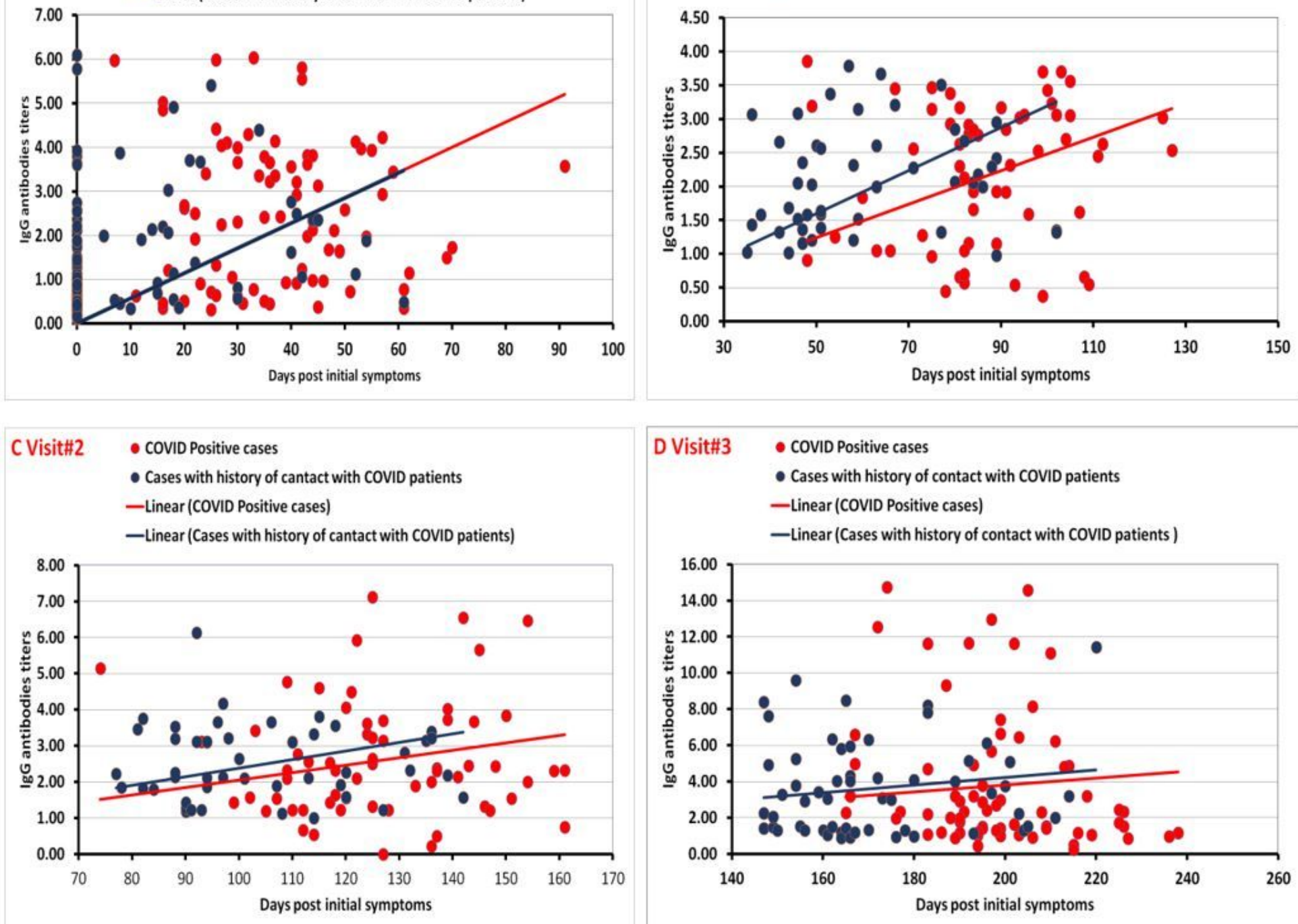

\section{Visit\#3 coVID Positive cases \\ - Cases with history of contact with COVID patients \\ - Linear (COVID Positive cases) \\ - Linear (Cases with history of contact with COVID patients)}

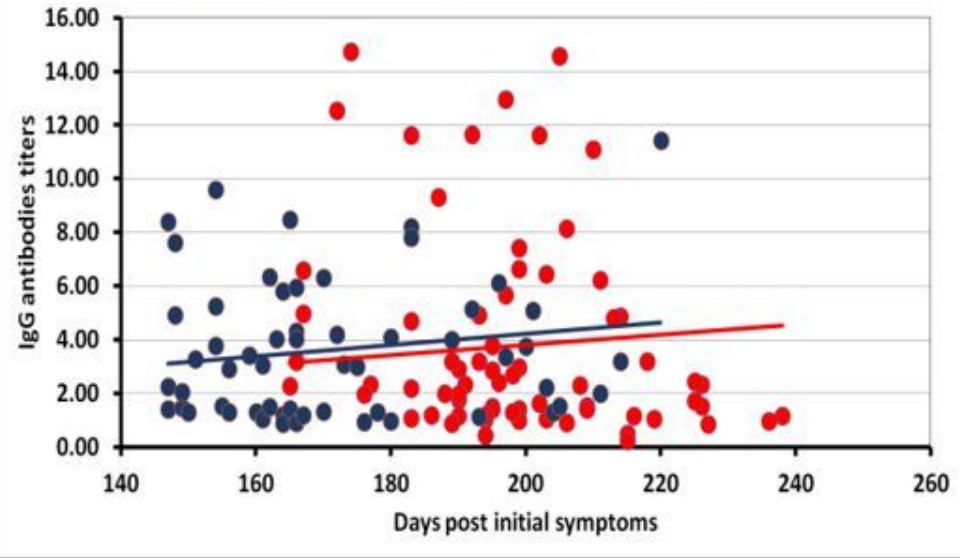

Figure 1

Correlation of dynamic changes in IgG antibody titers during follow-up of up to 32 weeks 
A

B

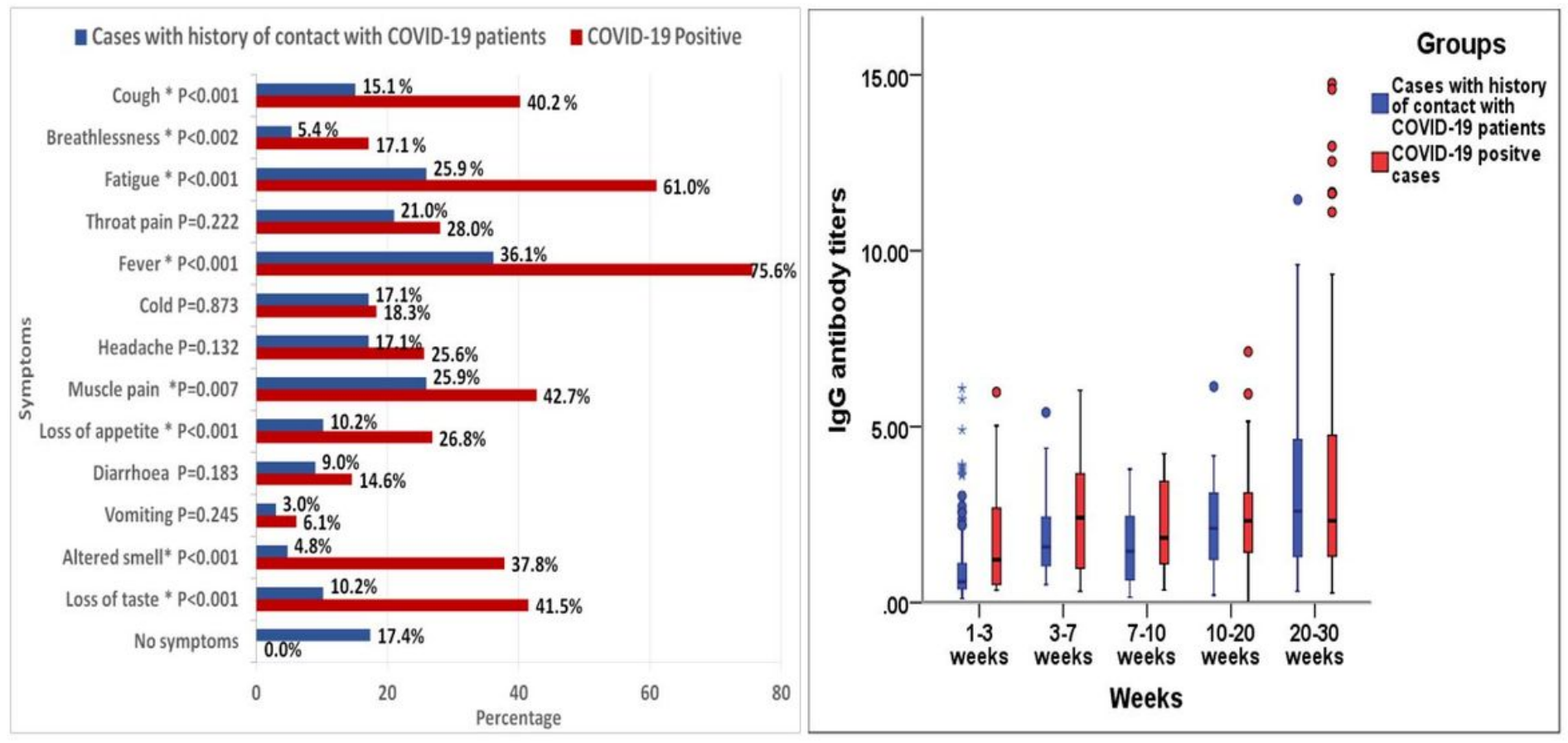

\section{Figure 2}

A. Frequency of symptoms among the two cohorts are shown. Symptoms with a significant difference $(P$ $<0.05)$ are marked with an asterisk. B. The optical density (OD) value of IgG antibodies against SARSCoV-2 in the study participants over the 32 weeks duration is shown. The median and quartiles were represented in the boxplots and the bars represent the maximum and minimum values. 